\title{
JOINT UNIVERSALITY OF HURWITZ ZETA-FUNCTIONS
}

\author{
ANTANAS LAURINČIKAS
}

\author{
(Received 29 September 2011)
}

\begin{abstract}
It is well known that Hurwitz zeta-functions with algebraically independent parameters over the field of rational numbers are universal in the sense that their shifts approximate simultaneously any collection of analytic functions. In this paper we introduce some classes of universal composite functions of a collection of Hurwitz zeta-functions.
\end{abstract}

2010 Mathematics subject classification: primary 11M41.

Keywords and phrases: limit theorem, space of analytic functions, Hurwitz zeta-function, universality.

\section{Introduction}

Let $s=\sigma+i t$ be a complex variable, and $\alpha, 0<\alpha \leq 1$, be a fixed parameter. The Hurwitz zeta-function $\zeta(s, \alpha)$ is defined, for $\sigma>1$, by

$$
\zeta(s, \alpha)=\sum_{m=0}^{\infty} \frac{1}{(m+\alpha)^{s}},
$$

and is meromorphically continued to the whole complex plane. The point $s=1$ is a simple pole, and $\operatorname{Res}_{s=1} \zeta(s, \alpha)=1$. For $\alpha=1$, the function $\zeta(s, \alpha)$ reduces to the Riemann zeta-function

$$
\zeta(s)=\sum_{m=1}^{\infty} \frac{1}{m^{s}}, \quad \sigma>1,
$$

and

$$
\zeta\left(s, \frac{1}{2}\right)=\left(2^{s}-1\right) \zeta(s)
$$

Properties of the function $\zeta(s, \alpha)$ depend on the arithmetical nature of the parameter $\alpha$. If $\alpha$ is transcendental or rational and not equal to 1 or $\frac{1}{2}$, then the function $\zeta(s, \alpha)$ is universal in the following sense. Let $K$ be a compact subset of the strip $D=\left\{s \in \mathbb{C}: \frac{1}{2}<\sigma<1\right\}$ with connected complement, and let $f(s)$ be a continuous

The author is partially supported by grant No. MP-94 from the Research Council of Lithuania.

(c) 2012 Australian Mathematical Publishing Association Inc. 0004-9727/2012 \$16.00 
function on $K$ which is analytic in the interior of $K$. Then, for every $\varepsilon>0$,

$$
\liminf _{T \rightarrow \infty} \frac{1}{T} \operatorname{meas}\left\{\tau \in[0, T]: \sup _{s \in K}|\zeta(s+i \tau, \alpha)-f(s)|<\varepsilon\right\}>0 .
$$

This was proved independently by Gonek [7] and Bagchi [1]; see also [6]. Here and in the following, meas $\{A\}$ denotes the Lebesgue measure of a measurable set $A \subset \mathbb{R}$. In the case $\alpha=1, \frac{1}{2}$, the function also remains universal; however, in this case, the function $f(s)$ must be nonvanishing on $K$. The case of algebraic irrational $\alpha$ is an open problem.

The first example of universal zeta-functions, the Riemann zeta-function $\zeta(s)$, was discovered by Voronin [20]. It is now known that the majority of zeta and $L$-functions are universal in the above sense; for history and references, see [1, 7-11, 14-16, 19].

Universality is a remarkable property of zeta- and $L$-functions having many applications. Universality is used in the investigation of functional independence, zero-distribution, value set denseness and other important problems in analytic number theory. A practical application of Voronin's universality theorem for estimation of some integrals is given in [4]. Therefore, the extension of the class of universal functions is an important problem in analytic number theory and theory of functions. In [13], we introduced some classes of functions $F$ such that the composite functions $F(\zeta(s))$ preserve the universality property. In the case of the Hurwitz zeta-function $\zeta(s, \alpha)$ with transcendental parameter $\alpha$, this is discussed in another paper ('On the universality of the Hurwitz zeta-function', submitted for publication).

Denote by $H(D)$ the space of analytic functions on $D$ endowed with the topology of uniform convergence on compacta. Among others, the following result in the paper just mentioned has been obtained. For $a_{1}, \ldots, a_{k} \in \mathbb{C}$, denote by $U_{H, a_{1}, \ldots, a_{k}}$ the class of continuous functions $F: H(D) \rightarrow H(D)$ such that $F(H(D)) \supset H_{a_{1}, \ldots, a_{k}}(D)$, where

$$
H_{a_{1}, \ldots, a_{k}}(D)=\left\{g \in H(D):\left(g(s)-a_{j}\right)^{-1} \in H(D), j=1, \ldots, k\right\} .
$$

Theorem 1.1. Suppose that the number $\alpha$ is transcendental, and $F \in U_{H, a_{1}, \ldots, a_{k}}$. For $k=1$, let $K \subset D$ be a compact subset with connected complement, and let $f(s)$ be a continuous function on $K$, not equal to $a_{1}$, and analytic in the interior of $K$. For $k \geq 2$, let $K \subset D$ be an arbitrary compact subset, and $f(s) \in H_{a_{1}, \ldots, a_{k}}(D)$. Then, for every $\varepsilon>0$,

$$
\liminf _{T \rightarrow \infty} \frac{1}{T} \operatorname{meas}\left\{\tau \in[0, T]: \sup _{s \in K}|F(\zeta(s+i \tau, \alpha))-f(s)|<\varepsilon\right\}>0 .
$$

For example, if $r=1$ and $a_{1}=0$, we have the universality of the function $e^{\zeta(s, \alpha)}$. If $r=2$ and $a_{1}=-1, a_{2}=1$, Theorem 1.1 implies the universality of the functions $\sin \zeta(s, \alpha)$ and $\cos \zeta(s, \alpha)$.

For zeta and $L$-functions, joint universality is also possible. In this case, a collection of analytic functions simultaneously is approximated uniformly on compact subsets of some region by shifts of zeta- or $L$-functions. The first result in this direction is also due to Voronin. In [21], he proved the joint universality of Dirichlet 
$L$-functions $L(s, \chi)$. Other versions of Voronin's theorem were given independently by Gonek [7] and Bagchi [1, 2]. We state a modern version of the Voronin theorem.

Theorem 1.2. Let $\chi_{1}, \ldots, \chi_{r}$ be pairwise nonequivalent Dirichlet characters. For $j=1, \ldots, r$, let $K_{j}$ be a compact subset of the strip $D$ with connected complement, and let $f_{j}(s)$ be a nonvanishing continuous function on $K_{j}$ which is analytic in the interior of $K_{j}$. Then, for every $\varepsilon>0$,

$$
\liminf _{T \rightarrow \infty} \frac{1}{T} \operatorname{meas}\left\{\tau \in[0, T]: \sup _{1 \leq j \leq r} \sup _{s \in K_{j}}\left|L\left(s+i \tau, \chi_{j}\right)-f_{j}(s)\right|<\varepsilon\right\}>0 .
$$

Hurwitz zeta-functions are also jointly universal. For $j=1, \ldots, r$, let $\alpha_{j} \in \mathbb{R}$, $0<\alpha_{j} \leq 1$, and $\zeta\left(s, \alpha_{j}\right)$ be the corresponding Hurwitz zeta-function. Assuming that the numbers $\alpha_{1}, \ldots, \alpha_{r}$ are algebraically independent over the field of rational numbers $\mathbb{Q}$, Nakamura [18] proved a joint universality theorem for the functions $\zeta\left(s, \alpha_{1}\right), \ldots, \zeta\left(s, \alpha_{r}\right)$. A slightly more general result by a different method was obtained in [12]. Let

$$
L\left(\alpha_{1}, \ldots, \alpha_{r}\right)=\left\{\log \left(m+\alpha_{j}\right): m \in \mathbb{N}_{0}=\mathbb{N} \cup\{0\}, j=1, \ldots, r\right\} .
$$

Theorem 1.3 [12]. Suppose that the set $L\left(\alpha_{1}, \ldots, \alpha_{r}\right)$ is linearly independent over $\mathbb{Q}$. For $j=1, \ldots, r$, let $K_{j}$ be a compact subset of the strip $D$ with connected complement, and let $f_{j}(s)$ be a continuous function on $K_{j}$ which is analytic in the interior of $K_{j}$. Then, for every $\varepsilon>0$,

$$
\liminf _{T \rightarrow \infty} \frac{1}{T} \operatorname{meas}\left\{\tau \in[0, T]: \sup _{1 \leq j \leq r} \sup _{s \in K_{j}}\left|\zeta\left(s+i \tau, \alpha_{j}\right)-f_{j}(s)\right|<\varepsilon\right\}>0 .
$$

If $\alpha$ is an algebraic irrational number, then, by the Cassels theorem [5], at least 51 percent of elements of the set

$$
\left\{\log (m+\alpha): m \in \mathbb{N}_{0}\right\}
$$

are linearly independent over $\mathbb{Q}$. Thus, in general, it is possible that Theorem 1.3 remains true even for some algebraic irrational numbers $\alpha_{1}, \ldots, \alpha_{r}$.

The aim of this paper is to consider the universality of $F\left(\zeta\left(s, \alpha_{1}\right), \ldots, \zeta\left(s, \alpha_{r}\right)\right)$ for some classes of the functions $F$.

One class of the functions $F$ with the universality property can be described as follows. We say that the function $F: H^{r}(D) \rightarrow H(D)$ belongs to the class $\operatorname{Lip}\left(\beta_{1}, \ldots, \beta_{r}\right), \beta_{1}>0, \ldots, \beta_{r}>0$, if the following hypotheses are satisfied.

1. For each polynomial $p=p(s)$, there exists an element $g \in F^{-1}\{p\} \subset H^{r}(D)$.

2. For every compact subset $K \subset D$ with connected complement, there exist a positive $c$, and compact subsets $K_{1}, \ldots, K_{r} \subset D$ with connected complements such that

$$
\sup _{s \in K}\left|F\left(g_{11}(s), \ldots, g_{1 r}(s)\right)-F\left(g_{21}(s), \ldots, g_{2 r}(s)\right)\right| \leq c \sup _{1 \leq j \leq r} \sup _{s \in K_{j}}\left|g_{1 j}(s)-g_{2 j}(s)\right|^{\beta_{j}}
$$

for all $\left(g_{j 1}, \ldots g_{j r}\right) \in H^{r}(D), j=1,2$. 
Theorem 1.4. Suppose that the set $L\left(\alpha_{1}, \ldots, \alpha_{r}\right)$ is linearly independent over $\mathbb{Q}$, and that $F \in \operatorname{Lip}\left(\beta_{1}, \ldots, \beta_{r}\right)$. Let $K \subset D$ be a compact subset with connected complement, and let $f(s)$ be a continuous function on $K$ which is analytic in the interior of $K$. Then, for every $\varepsilon>0$,

$$
\liminf _{T \rightarrow \infty} \frac{1}{T} \operatorname{meas}\left\{\tau \in[0, T]: \sup _{s \in K}\left|F\left(\zeta\left(s+i \tau, \alpha_{1}\right), \ldots, \zeta\left(s+i \tau, \alpha_{r}\right)\right)-f(s)\right|<\varepsilon\right\}>0 .
$$

Example 1.5. For $\left(g_{1}, \ldots, g_{r}\right) \in H^{r}(D)$, let

$$
F\left(g_{1}, \ldots, g_{r}\right)=c_{1} g_{1}^{\left(n_{1}\right)}+\cdots+c_{r} g_{r}^{\left(n_{r}\right)},
$$

where $c_{1}, \ldots, c_{r} \in \mathbb{C} \backslash\{0\}$ and $n_{1}, \ldots, n_{r} \in \mathbb{N}$. Then, clearly, for each polynomial $p(s)$, we have that $F^{-1} p(s) \in H^{r}(D)$. Thus, hypothesis 1 of the class $\operatorname{Lip}\left(\beta_{1}, \ldots, \beta_{r}\right)$ is satisfied.

Let $K \subset D$ be a compact subset with connected complement, and $K \subset G \subset K_{1}$, where $G$ is an open set and $K_{1} \subset D$ is a compact subset with connected complement. We take a simple closed contour $L$ lying in $K_{1} \backslash G$ and enclosing the set $K$. Then, by the Cauchy integral formula, we find that, for all $\left(g_{j 1}, \ldots, g_{j r}\right) \in H^{r}(D), j=1,2$ and $s \in K$,

$$
\begin{aligned}
\left|F\left(g_{11}(s), \ldots, g_{1 r}(s)\right)-F\left(g_{21}(s), \ldots, g_{2 r}(s)\right)\right| \\
\quad=\left|\sum_{j=1}^{r} c_{j} \frac{n_{j} !}{2 \pi i} \int_{L} \frac{g_{1 j}(z)-g_{2 j}(z)}{(z-s)^{n_{j}+1}} \mathrm{~d} z\right| \\
\quad \leq \sum_{j=1}^{r}\left|c_{j}\right| C_{j} \sup _{s \in L}\left|g_{1 j}(s)-g_{2 j}(s)\right| \\
\quad \leq \sum_{j=1}^{r}\left|c_{j}\right| C_{j} \sup _{s \in K_{1}}\left|g_{1 j}(s)-g_{2 j}(s)\right| \\
\quad \leq c \sup _{1 \leq j \leq r} \sup _{s \in K_{1}}\left|g_{1 j}(s)-g_{2 j}(s)\right|
\end{aligned}
$$

with some $C_{j}>0, j=1, \ldots, r$, and $c>0$. Thus, $F \in \operatorname{Lip}(1, \ldots, 1)$. In this case, $K_{1}=\cdots=K_{r}=K_{1}$.

We now present certain other classes of the functions $F$.

Theorem 1.6. Suppose that the set $L\left(\alpha_{1}, \ldots, \alpha_{r}\right)$ is linearly independent over $\mathbb{Q}$, and that $F: H^{r}(D) \rightarrow H(D)$ is a continuous function such that, for every open set $G \in H(D)$, the set $F^{-1} G$ is nonempty. Let $K$ and $f(s)$ be the same as in Theorem 1.4. Then the assertion of Theorem 1.4 is true.

Obviously, a function $F \in \operatorname{Lip}\left(\beta_{1}, \ldots, \beta_{r}\right)$ is continuous. Therefore, the hypotheses of Theorem 1.4 imply those of the next theorem. 
Theorem 1.7. Suppose that the set $L\left(\alpha_{1}, \ldots, \alpha_{r}\right)$ is linearly independent over $\mathbb{Q}$, and that $F: H^{r}(D) \rightarrow H(D)$ is a continuous function such that, for each polynomial $p=p(s)$, the set $F^{-1}\{p\}$ is nonempty. Let $K$ and $f(s)$ be the same as in Theorem 1.4. Then the assertion of Theorem 1.4 is true.

Let $H_{a_{1}, \ldots, a_{k}}$ be the same as above.

Theorem 1.8. Suppose that the set $L\left(\alpha_{1}, \ldots, \alpha_{r}\right)$ is linearly independent over $\mathbb{Q}$, and that $F: H^{r}(D) \rightarrow H(D)$ is a continuous function such that $F\left(H^{r}(D)\right) \supset H_{a_{1}, \ldots, a_{k}}(D)$. For $k=1$, let $K \subset D$ be a compact subset with connected complement, and let $f(s)$ be a continuous function on $K$, not equal to $a_{1}$, which is analytic in the interior of $K$. For $k \geq 2$, let $K \subset D$ be an arbitrary compact subset, and $f(s) \in H_{a_{1}, \ldots, a_{k}}(D)$. Then the assertion of Theorem 1.4 is true.

For example, if $k=1$ and $a_{1}=0$, we obtain that the function $\zeta^{n}\left(s, \alpha_{1}\right) p\left(\zeta\left(s, \alpha_{2}\right), \ldots\right.$, $\zeta\left(s, \alpha_{r}\right)$ ), where $p$ is a polynomial, $p \not \equiv 0$, and $n \in \mathbb{N} \backslash\{1\}$, is universal because the equation $g_{1}^{n} p\left(g_{2}, \ldots, g_{r}\right)=g$ with $g(s) \in H_{0}(D)$ always has a solution.

In the general case, the following statement holds true.

Theorem 1.9. Suppose that the set $L\left(\alpha_{1}, \ldots, \alpha_{r}\right)$ is linearly independent over $\mathbb{Q}$, and that $F: H^{r}(D) \rightarrow H(D)$ is a continuous function. Let $K \subset D$ be a compact subset, and $f(s) \in F\left(H^{r}(D)\right)$. Then the assertion of Theorem 1.4 is true.

Now let $M(D)$ denote the space of meromorphic functions on $D$ endowed with the topology of uniform convergence on compacta. Note that $H(D)$ is a subspace of $M(D)$.

Theorem 1.10. Suppose that the set $L\left(\alpha_{1}, \ldots, \alpha_{r}\right)$ is linearly independent over $\mathbb{Q}$, and that $F: H^{r}(D) \rightarrow M(D)$ is a continuous function. Let $K \subset D$ be a compact subset, and $f(s) \in F\left(H^{r}(D)\right) \cap H(D)$. Then the assertion of Theorem 1.4 is true.

For example, if the function $f(s) \in H_{0}(D)$, then it can be approximated by shifts of the function $\left(\zeta\left(s, \alpha_{1}\right) \cdots \zeta\left(s, \alpha_{r}\right)\right)^{-1}$. Moreover, any function $f(s) \in H(D)$ can be approximated by shifts of the function $\zeta^{\prime}\left(s, \alpha_{j}\right)\left(\zeta\left(s, \alpha_{1}\right) \cdots \zeta\left(s, \alpha_{r}\right)\right)^{-1}, j=1, \ldots, r$.

\section{Lemmas}

For the proof of the theorems on universality of composite functions (except Theorem 1.4), we will apply the probabilistic approach. Thus, we need limit theorems for probability measures in functional spaces as well as explicit forms for supports of the limit measures in them.

Denote by $\gamma$ the unit circle $\{s \in \mathbb{C}:|s|=1\}$ on the complex plane, and define

$$
\Omega=\prod_{m=0}^{\infty} \gamma_{m},
$$

where $\gamma_{m}=\gamma$ for all $m \in \mathbb{N}_{0}=\mathbb{N} \cup\{0\}$. By the Tikhonov theorem, with the product topology and pointwise multiplication, the infinite-dimensional torus $\Omega$ is a compact 
topological abelian group. Let

$$
\Omega^{r}=\Omega_{1} \times \cdots \times \Omega_{r}
$$

where $\Omega_{j}=\Omega$ for $j=1, \ldots, r$. Then $\Omega^{r}$ is also a compact topological abelian group. Therefore, on $\left(\Omega^{r}, \mathcal{B}\left(\Omega^{r}\right)\right)$, the probability Haar measure $m_{H}^{r}$ exists, and this gives a probability space $\left(\Omega^{r}, \mathcal{B}\left(\Omega^{r}\right), m_{H}^{r}\right)(\mathcal{B}(S)$ denotes the class of Borel sets of the space $S)$. Denote by $\omega_{j}(m)$ the projection of $\omega_{j} \in \Omega_{j}, j=1, \ldots, r$, to the coordinate space $\gamma_{m}, m \in \mathbb{N}_{0}$. For brevity, let $\omega=\left(\omega_{1}, \ldots, \omega_{r}\right) \in \Omega^{r}, \omega_{j} \in \Omega_{j}, j=1, \ldots, r$, and $\underline{\alpha}=\left(\alpha_{1}, \ldots, \alpha_{r}\right)$, and on the probability space $\left(\Omega^{r}, \mathcal{B}\left(\Omega^{r}\right), m_{H}^{r}\right)$ define the $H^{r}(D)$ valued random element $\zeta(s, \omega, \underline{\alpha})$ by the formula

$$
\zeta(s, \omega, \underline{\alpha})=\left(\zeta\left(s, \omega_{1}, \alpha_{1}\right), \ldots, \zeta\left(s, \omega_{r}, \alpha_{r}\right)\right),
$$

where

$$
\zeta\left(s, \omega_{j}, \alpha_{j}\right)=\sum_{m=0}^{\infty} \frac{\omega_{j}(m)}{\left(m+\alpha_{j}\right)^{s}}, \quad j=1, \ldots, r .
$$

Let $P_{\zeta}$ be the distribution of the random element $\zeta(s, \omega, \underline{\alpha})$, that is,

$$
P_{\zeta}(A)=m_{H}^{r}\left(\omega \in \Omega^{r}: \zeta(s, \omega, \underline{\alpha}) \in A\right), \quad A \in \mathcal{B}\left(H^{r}(D)\right) .
$$

We set, for $A \in \mathcal{B}\left(H^{r}(D)\right)$,

$$
P_{T}(A)=\frac{1}{T} \operatorname{meas}\{\tau \in[0, T]: \zeta(s+i \tau, \underline{\alpha}) \in A\},
$$

where

$$
\zeta(s, \underline{\alpha})=\left(\zeta\left(s, \alpha_{1}\right), \ldots, \zeta\left(s, \alpha_{r}\right)\right) .
$$

We now state a joint limit theorem in the space $H^{r}(D)$ for the vector $\zeta(s, \underline{\alpha})$.

Lemma 2.1. Suppose that the set $L\left(\alpha_{1}, \ldots, \alpha_{r}\right)$ is linearly independent over $\mathbb{Q}$. Then the measure $P_{T}$ converges weakly to $P_{\zeta}$ as $T \rightarrow \infty$.

The lemma is [12, Theorem 4].

For the following, we recall the following well-known fact from the theory of the weak convergence of probability measures. Let $S_{1}$ and $S_{2}$ be two metric spaces. A function $h: S_{1} \rightarrow S_{2}$ is called $\left(\mathcal{B}\left(S_{1}\right), \mathcal{B}\left(S_{2}\right)\right)$-measurable if, for every $A \in \mathcal{B}\left(S_{2}\right)$,

$$
h^{-1} A \in \mathcal{B}\left(S_{1}\right) .
$$

If $h$ is continuous, then it is $\left(\mathcal{B}\left(S_{1}\right), \mathcal{B}\left(S_{2}\right)\right)$-measurable. Suppose that the function $h$ is $\left(\mathcal{B}\left(S_{1}\right), \mathcal{B}\left(S_{2}\right)\right)$-measurable. Then every probability measure $P$ on $\left(S_{1}, \mathcal{B}\left(S_{1}\right)\right)$ induces the unique probability measure $P h^{-1}$ on $\left(S_{2}, \mathcal{B}\left(S_{2}\right)\right)$ defined by

$$
P h^{-1}(A)=P\left(h^{-1} A\right), \quad A \in \mathcal{B}\left(S_{2}\right) .
$$


Lemma 2.2. Suppose that $P_{n}, n \in \mathbb{N}$, and $P$ are probability measures on $\left(S_{1}, \mathcal{B}\left(S_{1}\right)\right)$, and the function $h: S_{1} \rightarrow S_{2}$ is continuous. If $P_{n}$ converges weakly to $P$ as $n \rightarrow \infty$, then $P_{n} h^{-1}$ also converges weakly to $P h^{-1}$ as $n \rightarrow \infty$.

A proof of the lemma can be found in [3].

Lemmas 2.1 and 2.2 imply the following statement.

Lemma 2.3. Suppose that the set $L\left(\alpha_{1}, \ldots, \alpha_{r}\right)$ is linearly independent over $\mathbb{Q}$, and that the function $F: H^{r}(D) \rightarrow H(D)$ is continuous. Then

$$
\frac{1}{T} \operatorname{meas}\{\tau \in[0, T]: F(\zeta(s+i \tau, \underline{\alpha})) \in A\}, \quad A \in \mathcal{B}(H(D)),
$$

converges weakly to $P_{\zeta} F^{-1}$ as $T \rightarrow \infty$.

A similar statement is also true in the space $M(D)$.

Lemma 2.4. Suppose that the set $L\left(\alpha_{1}, \ldots, \alpha_{r}\right)$ is linearly independent over $\mathbb{Q}$, and that the function $F: H^{r}(D) \rightarrow M(D)$ is continuous. Then

$$
\frac{1}{T} \operatorname{meas}\{\tau \in[0, T]: F(\zeta(s+i \tau, \underline{\alpha})) \in A\}, \quad A \in \mathcal{B}(M(D)),
$$

converges weakly to $P_{\zeta} F^{-1}$ as $T \rightarrow \infty$.

Let $S$ be a separable metric space, and $P$ be a probability measure on $(S, \mathcal{B}(S))$. Then a minimal closed set $S_{P} \subset S$ such that $P\left(S_{P}\right)=1$ is called the support of the measure $P$. The set $S_{P}$ consists of all elements $x \in S$ such that, for every open neighbourhood $G$ of $x$, the inequality $P(G)>0$ is satisfied.

The support of the distribution of an $S$-valued random element $X$ is called a support of $X$.

Lemma 2.5. Suppose that the set $L\left(\alpha_{1}, \ldots, \alpha_{r}\right)$ is linearly independent over $\mathbb{Q}$. Then the support of the measure $P_{\zeta}$ is the whole of $H^{r}(D)$.

The lemma is [12, Theorem 11].

Lemma 2.6. Under the hypotheses of Theorem 1.6, the support of the measure $P_{\zeta} F^{-1}$ is the whole of $H(D)$.

Proof. Let $g$ be an arbitrary element of the space $H(D)$. We take any open neighbourhood $G$ of $g$. Since the function $F$ is continuous, the set $F^{-1} G$ is also open, and, by a hypothesis of Theorem 1.6 , is nonempty. Thus, $F^{-1} G$ is an open neighbourhood of some element $\underline{g} \in H^{r}(D)$. Therefore, in view of Lemma 2.5,

$$
m_{H}^{r}\left(\omega \in \Omega^{r}: \zeta(s, \omega, \underline{\alpha}) \in F^{-1} G\right)>0 .
$$

This shows that

$$
m_{H}^{r}\left(\omega \in \Omega^{r}: F(\zeta(s, \omega, \underline{\alpha})) \in G\right)>0 .
$$

Since $g$ and $G$ are arbitrary, $H(D)$ is the support of the random element $F(\zeta(s, \omega, \underline{\alpha}))$, and is thus the support of $P_{\zeta} F^{-1}$. 
The case of Theorem 1.7 requires the Mergelyan theorem on the approximation of analytic functions by polynomials.

Lemma 2.7. Suppose that $K \subset \mathbb{C}$ is a compact subset with connected complement, and that $f(s)$ is a continuous function on $K$ which is analytic in the interior of $K$. Then, for every $\varepsilon>0$, there exists a polynomial $p(s)$ such that

$$
\sup _{s \in K}|f(s)-p(s)|<\varepsilon .
$$

A proof of the lemma is given in [17]; see also [22].

Lemma 2.8. Under the hypotheses of Theorem 1.7, the support of the measure $P_{\zeta} F^{-1}$ is the whole of $H(D)$.

Proof. There exists a sequence of compact subsets $\left\{K_{l}: l \in \mathbb{N}\right\} \subset D$ such that $K_{l} \subset K_{l+1}$, $l \in \mathbb{N}$,

$$
D=\bigcup_{l=1}^{\infty} K_{l},
$$

and if $K \subset D$ is a compact subset, then $K \subset K_{l}$ for some $l \in \mathbb{N}$; see, for example, [6]. For $g_{1}, g_{2} \in H(D)$, define

$$
\rho\left(g_{1}, g_{2}\right)=\sum_{l=1}^{\infty} 2^{-l} \frac{\sup _{s \in K_{l}}\left|g_{1}(s)-g_{2}(s)\right|}{1+\sup _{s \in K_{l}}\left|g_{1}(s)-g_{2}(s)\right|} .
$$

Then $\rho$ is a metric on $H(D)$ which induces the topology of uniform convergence on compacta. It is easily seen that $\rho\left(g_{1}, g_{2}\right)$ is small if

$$
\sup _{s \in K_{l}}\left|g_{1}(s)-g_{2}(s)\right|
$$

is small enough for the set $K_{l}$ with sufficiently large $l \in \mathbb{N}$. Therefore, approximation of functions in the space $H(D)$ reduces to that on the sets $K_{l}$. Clearly, we can choose the sets $K_{l}, l \in \mathbb{N}$, to be with connected complements, for example, we can take closed rectangles.

Let $g=g(s)$ be an arbitrary element of the space $H(D)$, and $G$ be any open neighbourhood of $g$. Suppose that $K \subset D$ is a compact subset with connected complement. Then by Lemma 2.7 , for every $\varepsilon>0$, there exists a polynomial $p=p(s)$ such that

$$
\sup _{s \in K}|g(s)-p(s)|<\varepsilon .
$$

Therefore, if the number $\varepsilon$ is small enough, we may assume that $p \in G$, too. Thus, by the hypotheses of Theorem 1.7 , the set $F^{-1} G$ is open and nonempty, therefore Lemma 2.5 implies that

$$
\begin{aligned}
P_{\zeta} F^{-1}(G) & =m_{H}^{r}\left(\omega \in \Omega^{r}: F(\zeta(s, \omega, \underline{\alpha})) \in G\right) \\
& =m_{H}^{r}\left(\omega \in \Omega^{r}: \zeta(s, \omega, \underline{\alpha}) \in F^{-1} G\right)>0 .
\end{aligned}
$$

Since $g$ and $G$ are arbitrary, then the support of $P_{\zeta} F^{-1}$ is the whole of $H(D)$. 
Lemma 2.9. Suppose that the set $L\left(\alpha_{1}, \ldots, \alpha_{r}\right)$ is linearly independent over $\mathbb{Q}$, and that $F: H^{r}(D) \rightarrow H(D)\left(F: H^{r}(D) \rightarrow M(D)\right)$ is a continuous function. Then the support of the measure $P_{\zeta} F^{-1}$ is the closure of $F\left(H^{r}(D)\right)$.

Proof. We argue in a manner similar to the proof of the above lemmas. Let $g$ be an arbitrary element of $F\left(H^{r}(D)\right)$, and $G$ be any open neighbourhood of $g$. Then there exists $g \in H^{r}(D)$ such that $F(g)=g$. Therefore, the set $F^{-1} G$ is an open neighbourhood of $\underline{g}$, and we have by Lemma 2.5 that

$$
P_{\zeta} F^{-1}(G)=P_{\zeta}\left(F^{-1} G\right)>0
$$

Moreover,

$$
P_{\zeta} F^{-1}\left(F\left(H^{r}(D)\right)\right)=P_{\zeta}\left(H^{r}(D)\right)=1 .
$$

Since the support is a closed set, this together with (2.1) proves the lemma.

For the proofs of universality theorems, we will use an equivalent of the weak convergence of probability measures in terms of open sets.

Lemma 2.10. Let $P_{n}, n \in \mathbb{N}$, and $P$ be probability measures on $(S, \mathcal{B}(S))$. Then $P_{n}$, as $n \rightarrow \infty$, converges weakly to $P$ if and only if, for every open set $G \subset S$,

$$
\liminf _{n \rightarrow \infty} P_{n}(G) \geq P(G)
$$

A proof of the lemma is given in [3, Theorem 2.1].

\section{Proof of theorems}

Proof of Theorem 1.4. By Lemma 2.7, there exists a polynomial $p=p(s)$ such that

$$
\sup _{s \in K}|f(s)-p(s)|<\frac{\varepsilon}{2} .
$$

In view of hypothesis 1 of the class $\operatorname{Lip}\left(\beta_{1}, \ldots, \beta_{r}\right)$, there exists an element $\left(g_{1}(s), \ldots, g_{r}(s)\right) \in F^{-1}\{p\} \subset H^{r}(D)$. Let $\tau \in \mathbb{R}$ satisfy the inequality

$$
\sup _{1 \leq j \leq r} \sup _{s \in K_{j}}\left|\zeta\left(s+i \tau, \alpha_{j}\right)-g_{j}(s)\right|<c^{-1 / \beta}\left(\frac{\varepsilon}{2}\right)^{1 / \beta},
$$

where $K_{1}, \ldots, K_{r} \subset D$ are compact subsets with connected complements corresponding to the set $K$ in hypothesis 2 of the class $\operatorname{Lip}\left(\beta_{1}, \ldots, \beta_{r}\right)$, and $\beta=\min _{1 \leq j \leq r} \beta_{j}$. Then by hypothesis 2 , for $\tau$ satisfying (3.2), we find that

$$
\begin{aligned}
& \sup _{s \in K} \mid F\left(\zeta\left(s+i \tau, \alpha_{1}\right), \ldots, \zeta\left(s+i \tau, \alpha_{r}\right)\right)-p(s) \mid \\
& \quad \leq c \sup _{1 \leq j \leq r} \sup _{s \in K_{j}}\left|\zeta\left(s+i \tau, \alpha_{j}\right)-g_{j}(s)\right|^{\beta_{j}} \leq c c^{-\beta / \beta}\left(\frac{\varepsilon}{2}\right)^{\beta / \beta}=\frac{\varepsilon}{2} .
\end{aligned}
$$


Thus, (3.2) and Theorem 1.3 imply the inequality

$$
\liminf _{T \rightarrow \infty} \frac{1}{T} \operatorname{meas}\left\{\tau \in[0, T]: \sup _{s \in K}|F(\zeta(s+i \tau, \underline{\alpha}))-p(s)|<\frac{\varepsilon}{2}\right\}>0 .
$$

This in combination with (3.1) shows that

$$
\liminf _{T \rightarrow \infty} \frac{1}{T} \text { meas }\left\{\tau \in[0, T]: \sup _{s \in K}|F(\zeta(s+i \tau, \underline{\alpha}))-f(s)|<\varepsilon\right\}>0,
$$

and the proof is complete.

Proof of Theorem 1.6. By Lemma 2.7, there exists a polynomial $p(s)$ such that (3.1) is satisfied. Define

$$
G=\left\{g \in H(D): \sup _{s \in K}|g(s)-p(s)|<\frac{\varepsilon}{2}\right\} .
$$

Then $G$ is an open neighbourhood of the polynomial $p(s)$. Since, by Lemma 2.6, polynomials are elements of the support of the measure $P_{\zeta} F^{-1}$, we have that $P_{\zeta} F^{-1}(G)>0$. Therefore, Lemmas 2.3 and 2.10 imply that

$$
\liminf _{T \rightarrow \infty} \frac{1}{T} \operatorname{meas}\{\tau \in[0, T]: F(\zeta(s+i \tau, \underline{\alpha})) \in G\} \geq P_{\zeta} F^{-1}(G)>0 .
$$

This and the definition of the set $G$ yield

$$
\liminf _{T \rightarrow \infty} \frac{1}{T} \operatorname{meas}\left\{\tau \in[0, T]: \sup _{s \in K}|F(\zeta(s+i \tau, \underline{\alpha}))-p(s)|<\frac{\varepsilon}{2}\right\}>0 .
$$

Combining this with (3.1) proves the theorem.

Proof of Theorem 1.7. We use the same arguments as in the proof of Theorem 1.6, and in place of Lemma 2.6 we apply Lemma 2.8 .

Proof of Theorem 1.8. We divide the proof into two cases, beginning with the case $k=1$. By Lemma 2.7 , there exists a polynomial $p(s)$ such that

$$
\sup _{s \in K}|f(s)-p(s)|<\frac{\varepsilon}{4} \text {. }
$$

Since $f(s) \neq a_{1}$ on $K$, we have that $p(s) \neq a_{1}$ on $K$ as well if $\varepsilon$ is small enough. Therefore, we can define a continuous branch of $\log \left(p(s)-a_{1}\right)$ which will be an analytic function in the interior of $K$. Thus, in view of Lemma 2.7 again, we can find a polynomial $p_{1}(s)$ such that

$$
\sup _{s \in K}\left|p(s)-a_{1}-e^{p_{1}(s)}\right|<\frac{\varepsilon}{4} .
$$

Clearly, the function $g_{1}(s) \stackrel{\text { def }}{=} e^{p_{1}(s)}+a_{1}$ is an element of $H(D)$, and $g_{1}(s) \neq a_{1}$. In other words, $g_{1}(s) \in H_{a_{1}}(D)$. Thus, by the hypothesis of the theorem and Lemma 2.9, $g_{1}(s)$ is 
an element of the support of the measure $P_{\zeta} F^{-1}$. Moreover, inequalities (3.3) and (3.4) show that

$$
\sup _{s \in K}\left|f(s)-g_{1}(s)\right|<\frac{\varepsilon}{2} \text {. }
$$

Define

$$
G_{1}=\left\{g \in H(D): \sup _{s \in K}\left|g(s)-g_{1}(s)\right|<\frac{\varepsilon}{2}\right\}
$$

Then $G_{1}$ is an open neighbourhood of $g_{1}(s)$, so $P_{\zeta} F^{-1}\left(G_{1}\right)>0$. This, Lemmas 2.3 and 2.10, and the definition of the set $G_{1}$ yield the inequality

$$
\liminf _{T \rightarrow \infty} \frac{1}{T} \operatorname{meas}\left\{\tau \in[0, T]: \sup _{s \in K}\left|F(\zeta(s+i \tau, \underline{\alpha}))-g_{1}(s)\right|<\frac{\varepsilon}{2}\right\}>0 .
$$

From this, using (3.5), we obtain the theorem in the case $k=1$.

We now turn to the case $k \geq 2$. Define

$$
G_{2}=\left\{g \in H(D): \sup _{s \in K}|g(s)-f(s)|<\varepsilon\right\} .
$$

Then, by Lemma 2.9 and the hypothesis of the theorem, the set $G_{2}$ is an open neighbourhood of an element $f(s)$ of the support of the measure $P_{\zeta} F^{-1}$. Therefore, by Lemmas 2.3 and 2.10 ,

$$
\begin{aligned}
& \liminf _{T \rightarrow \infty} \frac{1}{T} \operatorname{meas}\left\{\tau \in[0, T]: \sup _{s \in K}|F(\zeta(s+i \tau, \underline{\alpha}))-f(s)|<\varepsilon\right\} \\
& \geq P_{\zeta} F^{-1}\left(G_{2}\right)>0 .
\end{aligned}
$$

This completes the proof.

Proof of Theorem 1.9. We apply Lemma 2.9 and argue as in the proof of Theorem 1.8, in the case $k \geq 2$.

Proof of Theorem 1.10. Since $f(s) \in F\left(H^{r}(D)\right) \cap H(D)$, in view of Lemma 2.9, $f(s)$ is an element of the support of the measure $P_{\zeta} F^{-1}$. Therefore, the theorem follows from Lemmas 2.4 and 2.10 in the same way as Theorem 1.8 in the case $k \geq 2$.

\section{References}

[1] B. Bagchi, The Statistical Behaviour and Universality Properties of the Riemann Zeta-Function and Other Allied Dirichlet Series, PhD Thesis, Indian Statistical Institute, Calcutta, 1981.

[2] B. Bagchi, 'A joint universality theorem for Dirichlet $L$-functions', Math. Z. 181 (1982), 319-334.

[3] P. Billingsley, Convergence of Probability Measures (Wiley, New York, 1968).

[4] K. M. Bitar, N. N. Khuri and H. C. Ren, 'Path integrals and Voronin's theorem on the universality of the Riemann zeta-function', Ann. Phys. 211 (1991), 172-196.

[5] J. W. S. Cassels, 'Footnote to a note of Davenport and Heilbronn', J. Lond. Math. Soc. 36 (1961), 177-184.

[6] J. B. Conway, Functions of One Complex Variable (Springer, Berlin, 1973). 
[7] S. M. Gonek, Analytic Properties of Zeta and L-Functions, PhD Thesis, University of Michigan, 1979.

[8] K.-G. Grosse-Erdmann, 'Universal families and hypercyclic operators', Bull. Amer. Math. Soc. 36 (1999), 345-381.

[9] A. A. Karatsuba and S. M. Voronin, The Riemann Zeta-Function (de Gruyter, New York, 1992).

[10] A. Laurinčikas, Limit Theorems for the Riemann Zeta-Function (Kluwer Academic Publishers, Dordrecht, 1996).

[11] A. Laurinčikas, 'The universality of zeta-functions', Acta Appl. Math. 78 (2003), 251-271.

[12] A. Laurinčikas, 'The joint universality of Hurwitz zeta-functions', Siauliai Math. Semin. 3(11) (2008), 169-187.

[13] A. Laurinčikas, 'Universality of the Riemann zeta-function', J. Number Theory 130 (2010), 2323-2331.

[14] A. Laurinčikas and R. Garunkštis, The Lerch Zeta-Function (Kluwer Academic Publishers, Dordrecht, 2002).

[15] K. Matsumoto, 'Some problems on mean values and the universality of zeta and multiple zetafunctions', in: Analytic and Probab. Methods in Number Theory, Proc. 3rd Intern. Conf. (eds. A. Dubickas et al.) (TEV, Vilnius, 2002), pp. 195-199.

[16] K. Matsumoto, 'Probabilistic value-distribution theory of zeta-functions', Sugaku Expositions 17 (2004), 51-71.

[17] S. N. Mergelyan, 'Uniform approximations to functions of complex variable', in: Uspekhi Mat. Nauk, 7. 1952, pp. 31-122 (in Russian) $\equiv$ Amer. Math. Soc. Trans. 101 (1954), 99.

[18] T. Nakamura, 'The existence and nonexistence of joint $t$-universality for Lerch zeta-function', J. Number Theory $\mathbf{1 2 5}(2)$ (2007), 424-441.

[19] J. Steuding, Value Distribution of L-Functions, Lecture Notes in Mathematics, 1877 (Springer, Berlin, 2007).

[20] S. M. Voronin, 'Theorem on the "universality" of the Riemann zeta-function', Izv. Akad. Nauk SSSR, Ser. Matem. 39 (1975), 475-486 (in Russian) 三 Math. USSR Izv. 9 (1975), 443-453.

[21] S. M. Voronin, 'On the functional independence of Dirichlet $L$-functions', Acta Arith. 27 (1975), 493-503 (in Russian).

[22] J. L. Walsh, 'Interpolation and approximation by rational functions in the complex domain', Amer. Math. Soc. Colloq. Publ. 20 (1960).

\footnotetext{
ANTANAS LAURINČIKAS, Faculty of Mathematics and Informatics, Vilnius University, Naugarduko 24, LT-03225 Vilnius, Lithuania

e-mail: antanas.laurincikas@mif.vu.lt
} 